\title{
Chitosan Nanoparticles of 5-Fluorouracil for Ophthalmic Delivery: Characterization, in-Vitro and in-Vivo Study
}

\author{
Ramesh Chand Nagarwal, ${ }^{*, a}$ Paras Nath Singh, ${ }^{a}$ Shri Kant,${ }^{c}$ Pralay Maiti, ${ }^{b}$ and \\ Jayanta Kumar PANDIT *,a \\ ${ }^{a}$ Department of Pharmaceutics, Institute of Technology, Banaras Hindu University; ${ }^{b}$ School of Material Science \& \\ Technology, Institute of Technology, Banaras Hindu University; and ${ }^{c}$ Department of Ophthalmology, Institute of Medical \\ Sciences, Banaras Hindu University; Varanasi-221005, U.P, India.
}

Received October 14, 2010; accepted November 2, 2010; published online November 9, 2010

The aim of this investigation was to develop 5-fluorouracil (5-FU) loaded chitosan nanoparticles (CH-DNPs) for ophthalmic delivery. CH-DNPs were fabricated by ionotropic gelation mechanism using chitosan (CH) and a polyanion (TPP). The nanoparticles were smooth and spherical, confirmed by scanning electron microscopy (SEM) and atomic force microscope (AFM). CH/TPP mass ratio and TPP significantly changed the particles size morphology and encapsulation efficiency. The nanoparticles size ranged from approximately 114 to $192 \mathrm{~nm}$ and had a positive zeta potential $(30 \pm 4 \mathrm{mV})$. The encapsulation efficiency, loading capacity and recovery of DNPs were $8.12-34.32 \%, 3.14-15.24 \%$ and 24.22 to $67 \%$ respectively. Physical characterization was done by Fourier transform infrared (FT-IR) and X-ray diffraction (XRD). No interaction was observed in between drug and polymer and crystallinity of drug was not changed in drug loaded nanoparticles. In-vitro release study of DNPs showed diffusion controlled release. Bioavailability study of batch CS9 was studied in rabbit eye and compare to 5-FU solution. 5-FU level was significantly higher in aqueous humor of rabbit eye. Ocular tolerance was studied in the eye of New Zealand rabbits and tested formulation was non-irritant with no sign of inflammation.

Key words chitosan; nanoparticle; 5-fluorouracil; ophthalmic delivery; in-vitro; in-vivo

5-FU is a pyrimidine analogue commonly used to treat many epithelial cancers. It acts by interacting with $S$ phase cells (those actively synthesizing DNA). Therefore, it is suitable to treat squamous cell carcinoma because squamous tumours are composed of rapidly proliferating abnormal epithelial cells. It has limited side effects on the normal ocular surface epithelium. ${ }^{1)}$ 5-FU is an inexpensive drug, easily handled by medical personnel and patients, and is stable in aqueous solution for at least 3 weeks. It does not need to be stored in a refrigerator. Topical solution of this drug is always prepared extemporaneously. The acute and chronic side effects of mitomycin $\mathrm{C}(\mathrm{MMC})$ are definitely much more frequent and serious than those induced by 5-FU, as referred to by clinicians using MMC for pigmented conjunctival lesions. ${ }^{1)}$ Currently $1 \%$ of 5 -FU solution is used by the ophthalmologist which is a high concentration for ophthalmic application. Bioavailability at anterior segment of eye is obtained less than $5 \%$ of applied eye dose because of drainage and lacrimation and low retention exposure to the absorption surface.

Non ophthalmic nanoparticles of 5-FU using polymers such as poly(butylcyanoacrylate), ${ }^{2)}$ poly(lactic acid), poly(lactide-co-glycolide) $)^{3)}$ and chitosan ${ }^{4-6)}$ have been reported, but investigators have not explored the application of 5-FU loaded nanoparticles (DNPs) for the treatment of ocular application.

Ocular therapy by 5-FU can be improved and its toxicity diminished by facilitating the specific accumulation in the tumor infected regions with prolonged exposure of the cells to this agent. In this sense, the association of anticancer drugs to delivery systems has been an interesting approach for selectively delivering these agents and, at the same time, reducing their toxicity. Another benefit of 5-FU loaded nanoparticles in the targeted tissues could be an improvement in its pharmacokinetics profile.
Polymeric nanoparticulate systems have been evaluated as ocular drug delivery to enhance the absorption of therapeutic drugs to improve bioavailability, reduce side effects, and sustain intraocular drug levels. ${ }^{7)}$ In addition, chitosan $(\mathrm{CH})$ is suitable for fabrication of nanogel/nanoparticles of 5-FU because it is positively charged, making it able to adhere to the negatively charged ocular surface and is soluble in diverse acids and able to interact with polyanions to form complex and nanogel. The cornea and conjunctiva have negative charge so the mucoadhesive polymer might interact intimately with these structures and increase the concentration and residence time of the associated drug at the disease site. Among the mucoadhesive polymers, chitosan exhibits several favourable properties, such as biodegradability, nontoxicity, biocompatibility, and mucoadhesiveness. In fact, an ionic interaction between the positively charged amino groups of $\mathrm{CH}$ and negatively charged sialic acid residues in mucus has been proposed as the mucoadhesion mechanism. This unique combination of properties makes it a novel versatile biopolymer, which fulfils the requirement for its application in the ophthalmic field. ${ }^{8,9)}$

Based on these considerations, we hypothesized that a colloidal suspension of 5-FU loaded DNPs will increase the residence time of the drug in the precorneal area due to its mucoadhesive property and prolong the penetration of the drug into the intraocular structures, making it suitable the ophthalmic application.

In this work, we report the fabrication of 5-FU loaded chitosan nanoparticles (DNPs) and the particle size characterization by different techniques such as dynamic light scattering (DLS), scanning electron microscopy (SEM), and atomic force microscope (AFM). In-vitro release study was performed in phosphate buffer $(\mathrm{pH} 7.4)$ and Ex-in vivo study performed in fresh excised goat and rabbit eye. Bioavailability study was conducted in rabbit's eye. 


\section{Experimental}

Materials Chitosan ( $\mathrm{CH}$; medium viscosity grade) and sodium tripolyphosphate (TPP) were purchased from Sigma-Aldrich, U.S.A. 5-Fluorouracil (5-FU) was generously gifted by Dabur Pharma Ltd., India. Acetic acid, methanol and HPLC grade water were obtained from Merck India Ltd., Trehalose ( $\alpha$-D-gulcopyranosyl- $\alpha$-D-glucopyranoside) was obtained from C.D.H. Ltd., India. Other chemicals used were analytical grade.

Preparation of Nanoparticles Chitosan $(\mathrm{CH})$ nanoparticles were prepared by the ionotropic gelation method. ${ }^{10)}$ Blank nanoparticles were obtained by the addition of an aqueous solution of TPP to a $\mathrm{CH}$ solution in acetic acid ( $2 \% \mathrm{v} / \mathrm{v}, \mathrm{pH}$ adjusted to 4.5 with $0.1 \mathrm{~N} \mathrm{NaOH})$. Both TPP and $\mathrm{CH}$ were used at $0.1,0.15$ and $0.2 \% \mathrm{w} / \mathrm{v}$ concentrations. TPP was added to $\mathrm{CH}$ solution by flush method and stirred for $2 \mathrm{~h}$ at $500 \mathrm{rpm}$. The $5: 2$ ratio of $\mathrm{CH}$ : TPP was found to be optimum for the final batch. 5-FU loaded nanoparticles were obtained by the above described procedure and the ratios of $\mathrm{CH}$ and TPP remained unchanged. Different amounts $(0.05$ to $1 \% \mathrm{w} / \mathrm{v})$ of 5-FU were incorporated in the $\mathrm{CH}$ solution prior to the formation of nanoparticles in order to investigate the effect of the initial 5-FU concentration on the nanoparticle characteristics and in-vitro drug release. Nanoparticles were collected by cooling centrifugation (Beckman, Model J2-21, U.S.A.) at $18000 \mathrm{rpm}$ for $50 \mathrm{~min}$ at $4{ }^{\circ} \mathrm{C}$. Supernatant was subjected to analysis for unloaded drug concentration by HPLC at $266 \mathrm{~nm}$. The CH-DNPs pellet was suspended in triple distilled water using ultrasonication (UP50H, Hielscher Ultrasonics Gmbh, Germany) for $3 \mathrm{~min}$. The colloidal suspension was pre-frozen at $-80^{\circ} \mathrm{C}$ for $24 \mathrm{~h}$. D (+) Trehalose $(5 \%)$ was added as cryoprotectant to the colloidal suspension before the final freeze drying. DNPs were freeze dried $\left(-50^{\circ} \mathrm{C}\right)$ for $12 \mathrm{~h}$ by lyophilization (S.M Scientific Pvt. Ltd., New Delhi), and powder nanoaprticles were used for further characterization.

Nanoparticle Size and Morphology The size (Z-average mean) and zeta potential of the nanoparticles were analyzed by dynamic light scattering, in triplicate using a Zetasizer ZS (Malvern Instruments, U.K.). The nanosuspension of 5-FU was prepared with triple distilled water for size measurement.

Scanning electron microscopy (SEM) was performed using a FEI Quanta 200 ESEM FEG operating between 5 and $20 \mathrm{kV}$. Samples (nanosuspension) were deposited on a thin aluminum plate $\left(1 \times 1 \mathrm{~cm}^{2}\right)$ and dried at room temperature and directly placed on the stub without staining and focused at different magnifications (10000 to $100000 \times$ ).

A NT-MDT multimode atomic force microscope (AFM), Russia, controlled by a Solver scanning probe, was used for the surface morphology and three dimensional organization and/or association of the nanoparticles. Tapping mode was used with the tip mounted on a $100-\mu \mathrm{m}-\mathrm{long}$, single-beam cantilever with resonance frequency in the range of $240-255 \mathrm{kHz}$ and corresponding spring constant of $11.5 \mathrm{~N} / \mathrm{m}$. A drop of nanosuspension was deposited on a glass cover slip fixed on a metallic magnetic support. The drop was dried at $50^{\circ} \mathrm{C}$ in oven.

Encapsulation Efficiency and \% Recovery The fabricated nanoparticles suspension was centrifuged on a cooling centrifuge (Beckman, Model J2-21, U.S.A.) at $18000 \mathrm{rpm}$ at $4{ }^{\circ} \mathrm{C}$ for $50 \mathrm{~min}$. The amount of 5-FU was calculated as the difference between the total amount used to prepare the nanoparticles and amount present in the supernatant, the concentration of 5FU was determined by a HPLC method. Freeze dried CH-DNPs was used for determining drug loading capacity. 5-FU was extracted from DNP $(5 \mathrm{mg})$ with methanol $(20 \mathrm{ml})$ stirred at $500 \mathrm{rpm}$ at room temperature, and dispersion was ultrasonicated ( 60 magnitude, 0.8 cycles) for $3 \mathrm{~min}$ and filtered by $0.2 \mu \mathrm{m}$ membrane filter (Millipore, U.S.A.). The filtered solution was injected in the HPLC and drug concentration was calculated by calibration curve. The 5 -FU $\% \mathrm{EE}, \% \mathrm{LC}$ and $\% \mathrm{R}$ of the nanoparticles were determined in triplicate and calculated as follows.

$$
\begin{aligned}
& \text { encapsulation efficiency }(\% \mathrm{EE}) \\
& =[(\text { total amount of } 5-\mathrm{FU}-\text { free } 5 \text {-FU }) / \text { total } 5 \text {-FU }] \times 100 \\
& \text { drug loading capacity }(\% \mathrm{LC}) \\
& =[(\text { total amount of } 5 \text {-FU/total weight of } \mathrm{NPs}) \times 100 \\
& \text { recovery }(\% \mathrm{R})= \\
& \text { (total weight of nanoparticles/ } \\
& \text { total weight of polymer, drug and other excipients }) \\
& \quad \times 100
\end{aligned}
$$

Drug-Polymer Interaction Study DNPs and blank NPs were separated by centrifugation from the nanosuspension and freeze dried. FT-IR spectra were obtained using a $\mathrm{KBr}$ pellet in FT-IR spectrophotometer (Shimadzu$8400 \mathrm{~S}$, Japan). \%Transmittance (\%T) was recorded in the spectral region of $500-4500 \mathrm{~cm}^{-1}$ using a resolution of $4 \mathrm{~cm}^{-1}$ and 40 scans.
The XRD measurements were carried out using Bruker D8 Advance Xray diffractometer. The $\mathrm{X}$-rays were produced using a sealed tube and the wavelength of X-ray was $0.154 \mathrm{~nm}(\mathrm{Cu} K \alpha)$. The X-rays were detected using a fast counting detector based on Silicon strip technology (Bruker LynxEye dtector).

Interaction Study of CH-DNPs with the Mucin The in-vitro interaction of CH-DNPs with mucin ('porcine stomach type II' from Sigma Aldrich) was measured by viscosity change on Brookfield viscometer using the spindle 63 at $37^{\circ} \mathrm{C}$.

In-Vitro Release Studies In-vitro release studies of 5-FU loaded nanoparticles were performed by dialysis membrane (Himedia Ltd., India) with a molecular weight cut-off of 12000-14000. The membrane opening was tied to the opening of a polyvinyl test tube $(1 \mathrm{~cm}$ diameter $)$ and dipped in a $100 \mathrm{ml}$ beaker containing phosphate buffer $(\mathrm{pH} 7.4,50 \mathrm{ml})$. The entire system was placed in a larger beaker $(250 \mathrm{ml})$ containing distilled water used as outer jacket to maintain the temperature of medium at $37 \pm 0.5^{\circ} \mathrm{C}$. A small magnetic bead (Sigma-Aldrich, U.S.A.) was placed in the beaker and was stirred at $100 \mathrm{rpm}$ on a magnetic stirrer (Remi India Ltd.). At predetermined periods, $500 \mu \mathrm{l}$ of the medium was removed and the amount of 5-FU was analyzed by HPLC.

In Vivo Study In-vivo experiments were performed on groups of three either male or female New Zealand albino rabbits model (Central animal house, Institute of Medical Sciences (IMS), Banaras Hindu University (BHU), Varanasi, India) weighing from 2.1 to $2.5 \mathrm{~kg}$, free of any signs of inflammation or gross abnormality. All experiments were conducted with the permission of Central Animal Ethical Committee, IMS, BHU, Varanasi. Each conscious animal received 4 instillation of $50 \mu \mathrm{l}$ of sterile $0.1 \% 5$-FU solution and the optimized batch CS9 in the Cul-de-sac of the right eye at 5 min intervals, while normal saline was instilled in the left eye as control. After last instillation rabbits were maintained in an upright position using restraining boxes. After $0.5,1,2,4$, and $6 \mathrm{~h}, 50 \mu \mathrm{l}$ aqueous humor was withdrawn after anaesthetized by $20 \mathrm{mg} / \mathrm{kg}$ of ketamine hydrochloride (Aneket ${ }^{\mathbb{B}}$, Neon Laboratories Ltd., Mumbai, India) and one drop of local anesthetic ( $0.5 \%$ proparacaine hydrochlodide). Aqueous humor was collected with 26 gauge needle attached to a tuberculin syringe. Each eye was examined after taking samples for any damage to iris, lens and cornea using a slit lamp. The Zinc sulphate $(2 \% \mathrm{w} / \mathrm{v})$ solution was added to samples to precipitate the protein and separated by cooling centrifuge at $15000 \mathrm{rpm}$ at $4{ }^{\circ} \mathrm{C}$ for $15 \mathrm{~min}$. Supernatant were filtered by $0.22 \mu \mathrm{m}$ membrane filter and analyzed by HPLC. After 2, 4, and $6 \mathrm{~h}$ vitreous humor and blood samples were also collected to see concentration in the back of eye and processed by the above procedure. Tolerability of with or without drug loaded nanoparticles were tested by Draize test using rabbit model ${ }^{12)}$ and the concession, swelling and discharge of the conjunctiva were graded on a scale from 0 to 3,0 to 4 , and 0 to 3 , respectively. Corneal integrity was checked by staining methylene blue.

Analysis of 5-FU by HPLC The assay of 5-FU was performed by HPLC using a high precision pump (Model CE4201, Cecil U.K.), a UV detector and a reverse phase column (Hamilton, HxSil C18 $5 \mu \mathrm{m} 150 \times 4.6$ $\mathrm{mm}$, U.S.A.). The mobile phase was $90 \% \mathrm{v} / \mathrm{v}$ water and $10 \% \mathrm{v} / \mathrm{v}$ of methanol. The 5-FU concentration range used to construct calibration curve was from 0.005 to $0.1 \mathrm{mg} / \mathrm{ml}$. The flow rate was $1 \mathrm{ml} / \mathrm{min}$. A $20 \mu 1$ sample was injected by a micro syringe (Hamilton, U.S.A.) and the eluent was monitored for the absorbance of 5-FU by UV detector at $266 \mathrm{~nm} .{ }^{11)}$

Stability Studies 5-FU loaded nanoparticles (DNPs) were placed in capped vials and sterilized by autoclaving and stored at controlled temperatures viz. $4 \pm 1{ }^{\circ} \mathrm{C}$, room temperature $\left(25 \pm 1^{\circ} \mathrm{C}\right)$, and $45 \pm 1^{\circ} \mathrm{C}$ for a period of 3 and 6 months with or without preservatives (benzalkonium chloride $0.01 \%)$. The formulations were periodically evaluated for drug content, $\mathrm{pH}$, microbial growth and chemical stability by FT-IR.

Data Analysis The differences in average of data were compared by simple analysis of variance (one-way ANOVA) or independent sample $t$-test (Origin 6.1 U.S.A.). The significance of the difference was determined at $95 \%$ confidence limit $(p<0.05)$.

\section{Results}

Particle Size and Morphology of Nanoparticles The size range of designed batches (CS1_CS9) was between 114 to $192 \mathrm{~nm}$ (Table 1). The encapsulation efficiency and zeta potential both were affected by the particle size and its distribution. The average zeta potential of DNPs and NPs were found $30 \pm 8$, and $42 \pm 5 \mathrm{mV}$ respectively. At different TPP 
concentrations $(0.1,0.2,0.3,0.4,0.5 \%)$ and $0.15 \% \mathrm{w} / \mathrm{v}$ of $\mathrm{CH}$, nanoparticles were obtained in the size range of 50.75 to $531.2,43.82$ to $955.4,37.84$ to $615.1,28.2$ to 955.4 and 32.67 to 1484 diameter nm respectively (Fig. 1). The results showed narrow size distribution at $0.1 \%$ TPP and wider distribution at $0.5 \%$ TPP.

SEM and AFM images of the $\mathrm{CH}$ nanoparticles were spherical in shape with smooth surface and in a narrow size range $(50-255 \mathrm{~nm})$ (Fig. 2) indicated that size distribution

Table 1. Formulation Composition and Characterization of DNPs $(n=3)$

\begin{tabular}{ccccccc}
\hline \hline $\begin{array}{c}\text { Batch } \\
\text { code }\end{array}$ & $\begin{array}{c}\mathrm{CH} \\
(\% \mathrm{w} / \mathrm{v})\end{array}$ & $\begin{array}{c}\mathrm{TPP} \\
(\% \mathrm{w} / \mathrm{v})\end{array}$ & $\begin{array}{c}\mathrm{PP} \\
(\mathrm{nm} \pm \mathrm{S} . \mathrm{D} .)\end{array}$ & $\% \mathrm{EE} \pm$ S.D. $\% \mathrm{HC} \pm \mathrm{S} . \mathrm{D}$. & $\% \mathrm{R} \pm \mathrm{S} . \mathrm{D}$. \\
\hline CS1 & 0.1 & 0.1 & $114 \pm 3$ & $8.1 \pm 1.2$ & $3.1 \pm 0.9$ & $24 \pm 2.1$ \\
CS2 & 0.1 & 0.15 & $122 \pm 4$ & $14 \pm 1.5$ & $5.2 \pm 1.1$ & $30 \pm 1.6$ \\
CS3 & 0.1 & 0.20 & $127 \pm 5$ & $18 \pm 1.1$ & $7.1 \pm 1.2$ & $51 \pm 1.9$ \\
CS4 & 0.15 & 0.1 & $144 \pm 3$ & $16 \pm 2.1$ & $9.4 \pm 1.5$ & $38 \pm 2.2$ \\
CS5 & 0.15 & 0.15 & $153 \pm 2$ & $25 \pm 1.9$ & $19 \pm 1.1$ & $47 \pm 2.4$ \\
CS6 & 0.15 & 0.2 & $178 \pm 5$ & $32 \pm 2.2$ & $21 \pm 1.7$ & $56 \pm 1.9$ \\
CS7 & 0.2 & 0.1 & $168 \pm 3$ & $26 \pm 2.8$ & $6.4 \pm 1.4$ & $46 \pm 2.5$ \\
CS8 & 0.2 & 0.15 & $183 \pm 4$ & $31 \pm 2.4$ & $13 \pm 1.8$ & $56 \pm 3.1$ \\
CS9 & 0.2 & 0.2 & $192 \pm 6$ & $34 \pm 2.9$ & $15 \pm 1.7$ & $67 \pm 2.3$ \\
\hline
\end{tabular}

$\mathrm{CH}$ : chitosan, \%EE: encapsulation efficiency; \%LC: loading capacity; \%R: recovery: PP: particle size $5: 2$ ratio of $\mathrm{CH}$ : TPP for all batches.

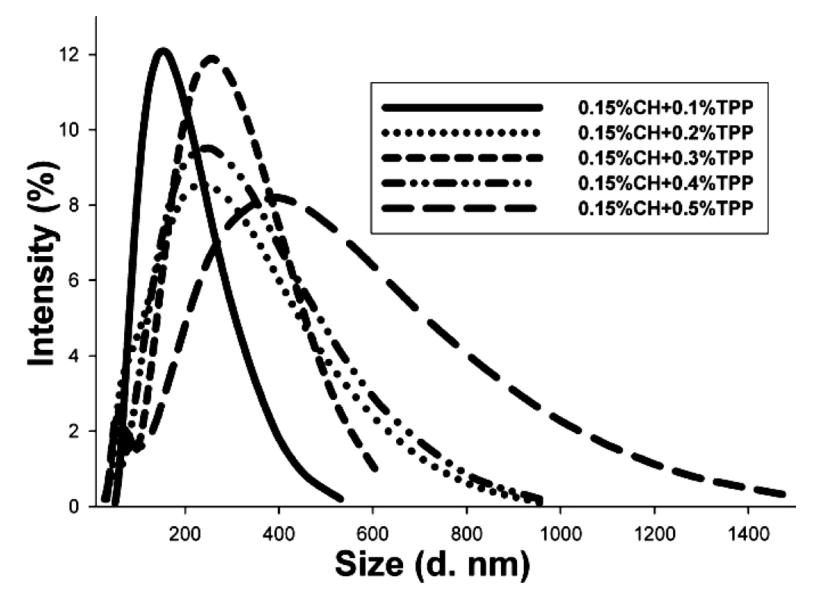

Fig. 1. Effect of TPP Concentration on Average Particle Size and Its Distribution (Plotted by DLS Data)

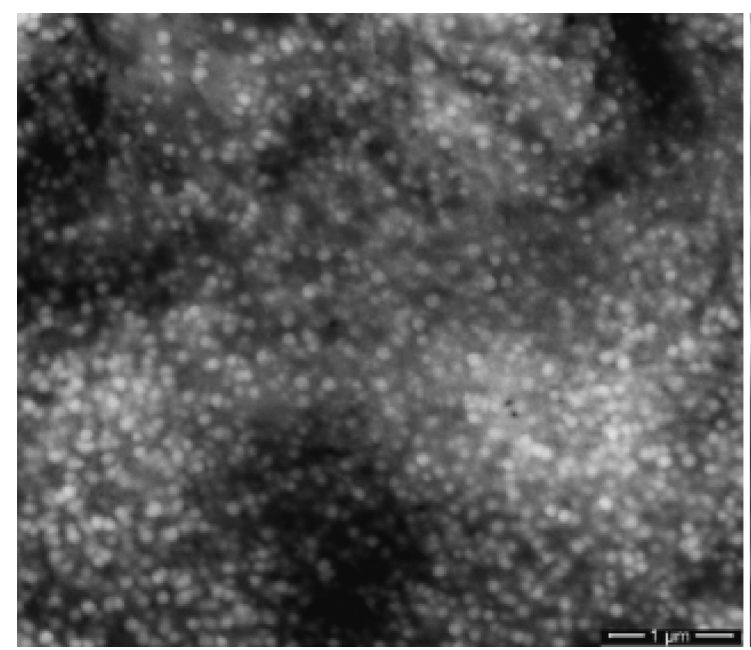

of the particles was at variance as compared to results of DLS. Some larger particles were also observed. 2D and 3D images of nanoparticles were recorded by AFM (Fig. 3) and size of individual particles was measured by software Nova and nanoparticles were rounded with smooth surface.

Effect of Mass Ratio of CH and TPP The size of the nanoparticles was found to have altered at different $\mathrm{CH}$ : TPP mass ratios. Increasing $\mathrm{CH}$ : TPP mass ratio increased the size of the nanoparticles (Fig. 4a). The mass ratio was critical and controls the size and size distribution of the nanoparticles. It can be seen from Fig. 4a that by decreasing the $\mathrm{CH}$ : TPP mass ratio, nanoparticles with smaller sizes $(\mathrm{nm})$ were produced. The 5:2 mass ratio was selected to design batches to study the effect of different concentrations of $\mathrm{CH}$ and TPP on particle size, encapsulation efficiency of 5-FU, loading capacity and recovery of nanoparticles (Table 1).

Effect of CH and TPP on Particle Size and Drug Loading Efficiency Narrow size distribution was obtained and spherical shape and smaller size of particles were observed (Fig. 2). Different combinations $(0.1,0.15,0.2 \% \mathrm{w} / \mathrm{v})$ of $\mathrm{CH}$ and TPP $(0.1,0.15,0.2 \% \mathrm{w} / \mathrm{v})$ were tried. The effect of both $\mathrm{CH}$ and TPP were studied on particle size and drug encapsulation efficiency for final optimization of the formulation. In the first trial $\mathrm{CH}$ concentration $(0.1,0.15,0.2 \% \mathrm{w} / \mathrm{v})$ was varied with fixed concentration of TPP. Particle size was obtained in increasing order with increasing $\mathrm{CH}$ concentration (Table 1). Same trend was also observed with variation of TPP concentration $(0.1$ to $0.5 \% \mathrm{w} / \mathrm{v})$ at fixed concentration of $\mathrm{CH}$ (Fig. 4b). Overall the particles were obtained in the size range 114.1 to $191.7 \mathrm{~d} \cdot \mathrm{nm}$. Encapsulation efficiency of $18.46,34.46$ and $44.32 \%$ were obtained at $0.1 / 0.2,0.15 / 0.2$ and $0.2 / 0.2 \% \mathrm{w} / \mathrm{v}$ mass ratio of $\mathrm{CH}$ : TPP respectively. Encapsulation efficiency increased with increase in $\mathrm{CH}$ concentration from 0.1 to $0.2 \% \mathrm{w} / \mathrm{v}$, but further decreased the encapsulation efficiency (data not shown). It may be due to increasing solution viscosity with higher concentration of $\mathrm{CH}$. It has been already reported that high viscosity associated with increased $\mathrm{CH}$ concentration hinders entrapment of 5-FU by preventing movement of 5-FU around the polymer chain.

Effect of pH and Drug Concentration on Encapsulation Efficiency Increasing the concentration of 5-FU significantly changed the size of the nanoparticles, due to in-

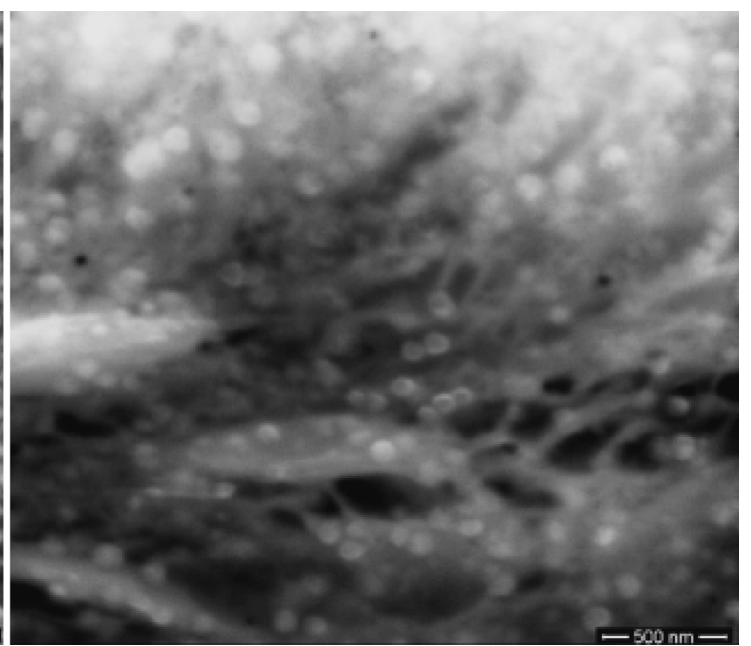

Fig. 2. SEM Images at Different Magnification (A: $50000 \times$, B: $100000 \times)$ of $\mathrm{CH}$ Nanoparticles $(0.15 \% \mathrm{CH} / 0.15 \%$ TPP; $5: 2 \mathrm{Ratio})$ 
creasing drug encapsulation efficiency (Fig. 4c). Drug loading efficiency was also increased (19.68 to $33.65 \%$ ) with increasing concentration of 5-FU $(0.05$ to $1 \% \mathrm{w} / \mathrm{v})($ Fig. $4 \mathrm{~d})$. The $\mathrm{pH}$ significantly $(p>0.05)$ affected the drug loading efficiency. Percentage loading was increased at higher $\mathrm{pH}$ but simultaneously particle size also increased with increase in $\mathrm{pH}$ of $\mathrm{CH}$ solution. It may be due to ionization of the drug at higher $\mathrm{pH}$. The particle size and surface charge were optimum at $\mathrm{pH}$ 4.5. The size was increased at higher $\mathrm{pH}$ (5.5 to 6.5) and which might be caused by the formation of the linkage or aggregation of the nanoparticles.
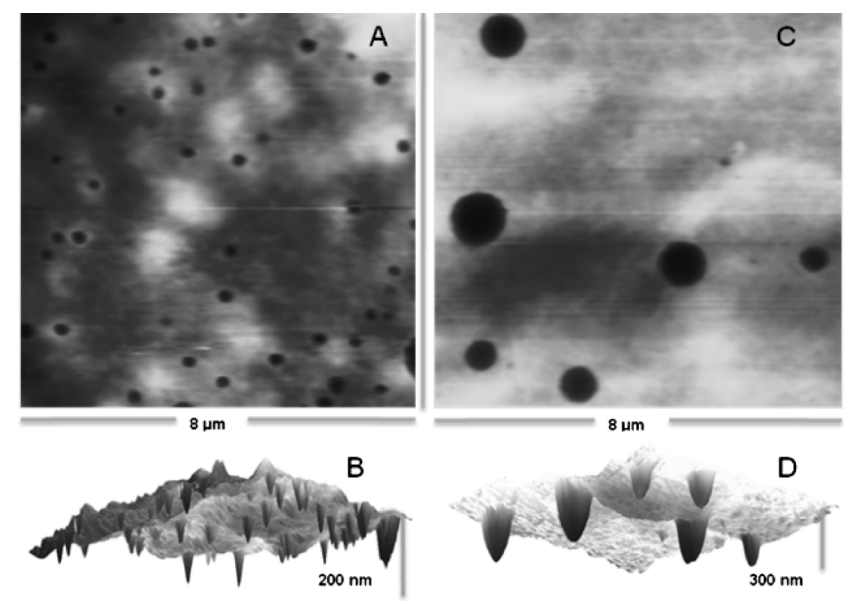

Fig. 3. (A, C) AFM Microimages of $\mathrm{CH}$ Nanoparticles $(0.15 \% \mathrm{CH} / 0.15 \%$ TPP; $5: 2$ Ratio), (B, D) 3D Images of NPs

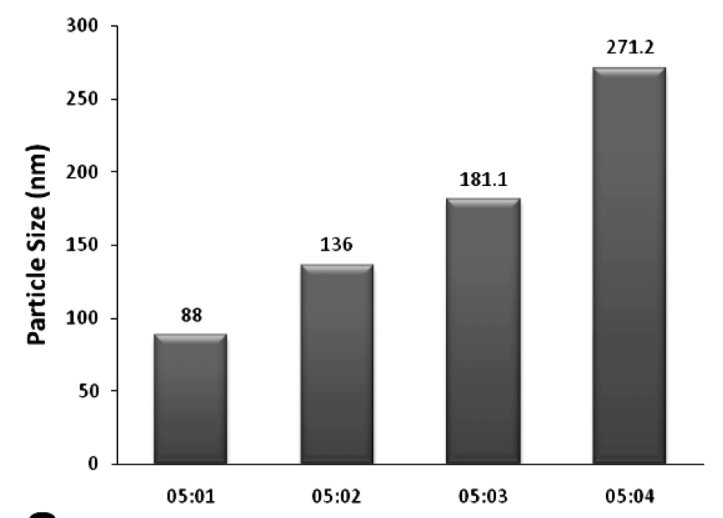

a

CH:TPP Mass Ratio

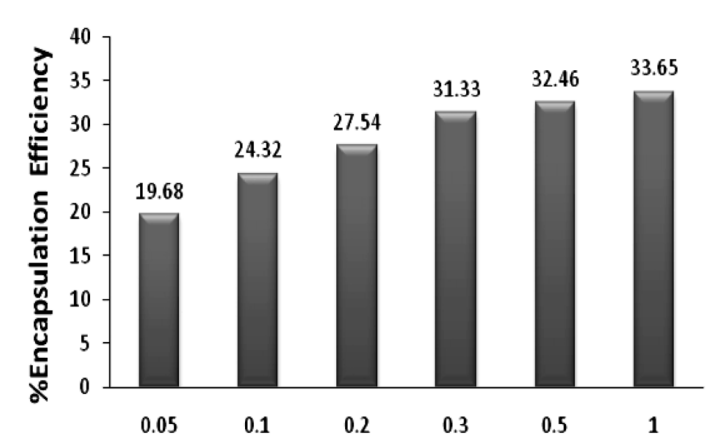

C

Drug Concentration $(\% \mathrm{w} / \mathrm{v})$
Effect of Swelling on Particle Size and Stability of Nanosuspension Nanoparticles were dispersed in buffer medium (pH 7.4) and stored for 3 and 6 month periods. Particle size was measured after 2 weeks and one and three month, the stored particles were found bigger in size and lost their spherical shape because of swelling (Fig. 5) as compared to the average size of freshly prepared nanoparticles. It is supported by AFM images, in Fig. 4 particles were sphere shaped but it become irregular shape in Fig. 5).

Stored formation in vials was tested for average particles size by dynamic light scattering after 1, 2, 3, 6 months and the size was increased due to water uptake and swelling behavior of chitosan. The $\mathrm{pH}$ was slightly changed and no color change was found. Small lumps of particles were found that may be particle aggregation or due to microbial growth after six month. No microbial growth was found in vial contained preservative. No additional peak was found in FT-IR spectra of stored powder for 3 and 6 month so it concludes the no chemical modification was found.

FT-IR and XRD of CH-DNPs Figure 6 shows the FTIR spectra of $\mathrm{CH}, 5-\mathrm{FU}$, blank NPs and DNPs. There are three characteristic peaks of $\mathrm{CH}$ at $3422 \mathrm{~cm}^{-1}$ of $v(\mathrm{OH})$, $1075 \mathrm{~cm}^{-1}$ of $v(\mathrm{C}-\mathrm{O}-\mathrm{C})$, and $1637 \mathrm{~cm}^{-1}$ of $v\left(\mathrm{NH}_{2}\right)$. The spectrum of CH-TPP (blank NPs) was different from that of $\mathrm{CH}$. In CH-TPP (NPs) the peak becomes wider, indicating more hydrogen bonding. The amino group transmittance is shifted at $1645 \mathrm{~cm}^{-1}$, which is an indication that these groups interacted with TPP creating ionic bonds. These interactions reduce the solubility of $\mathrm{CH}$ and are responsible for nanoparticle formation. In the DNPs the peak at $3417 \mathrm{~cm}^{-1}$ indicates
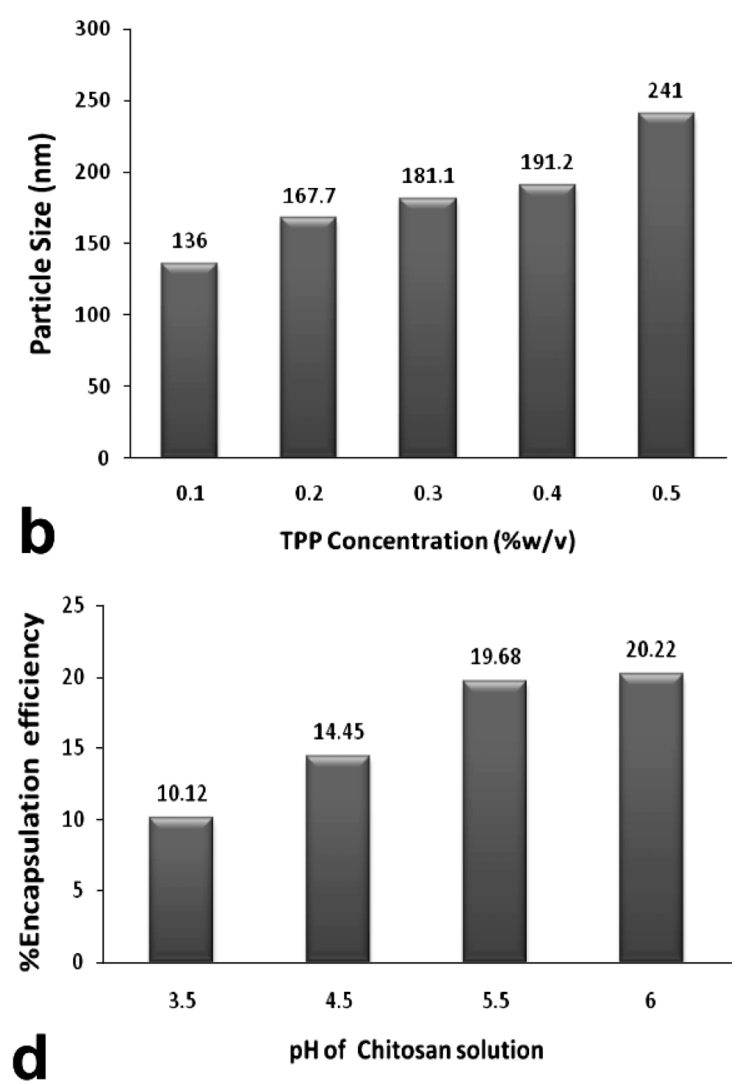

Fig. 4. (a) Effect of CH/TPP Mass Ratio on Particle Size of NPs; (b) Effect of TPP Concentration on Particles Size (d·nm) of CH Nanoparticles; (c) Effect of 5-FU Concentration on \% EE of DNPs (0.15\% CH : $0.15 \%$ TPP; $5: 2$ Ratio); (d) Effect of pH of CH Solution on \% EE of DNPs (0.15\% CH: $0.15 \%$ TPP; $5: 2$ Ratio) 


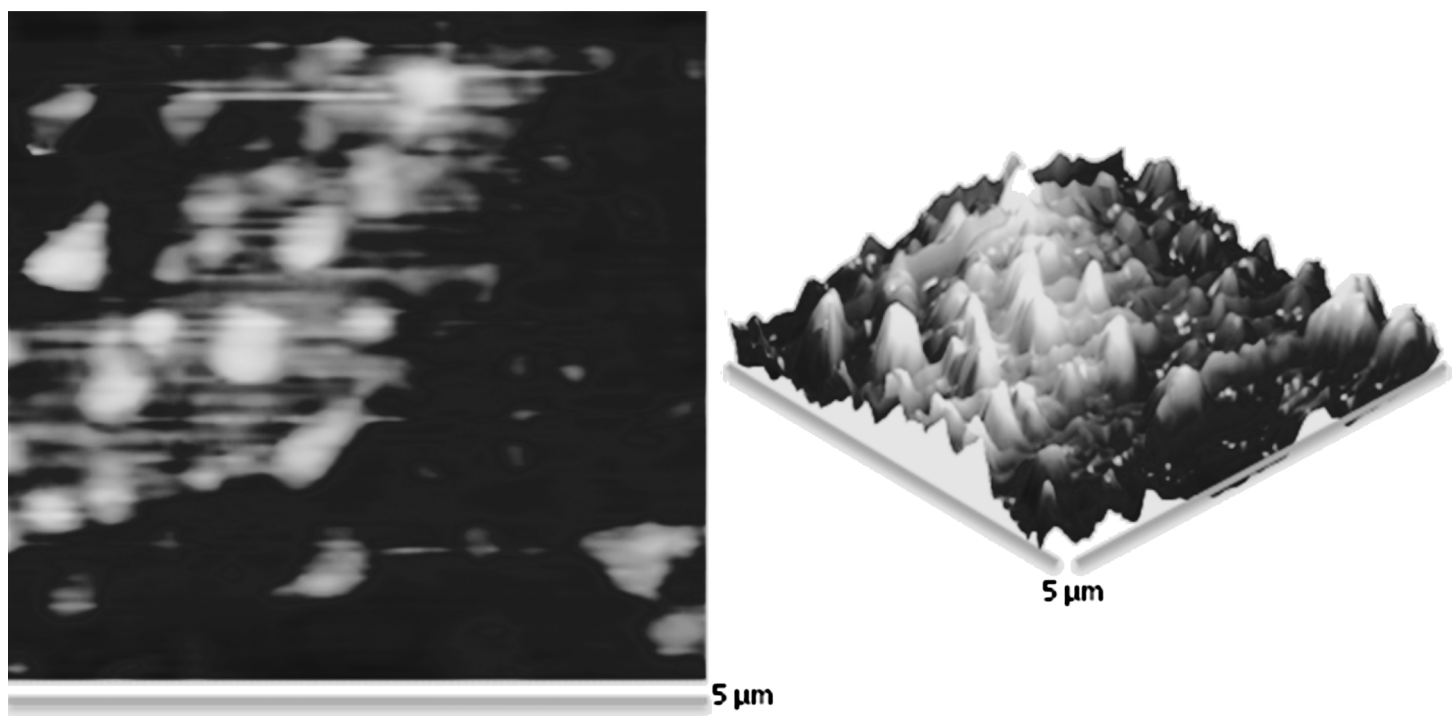

Fig. 5. AFM Micro Image of $\mathrm{CH}$ Nanoparticles after Storage as Nanosuspension for 1 Month at Room Temperature $\left(27 \pm 10{ }^{\circ} \mathrm{C}\right)$

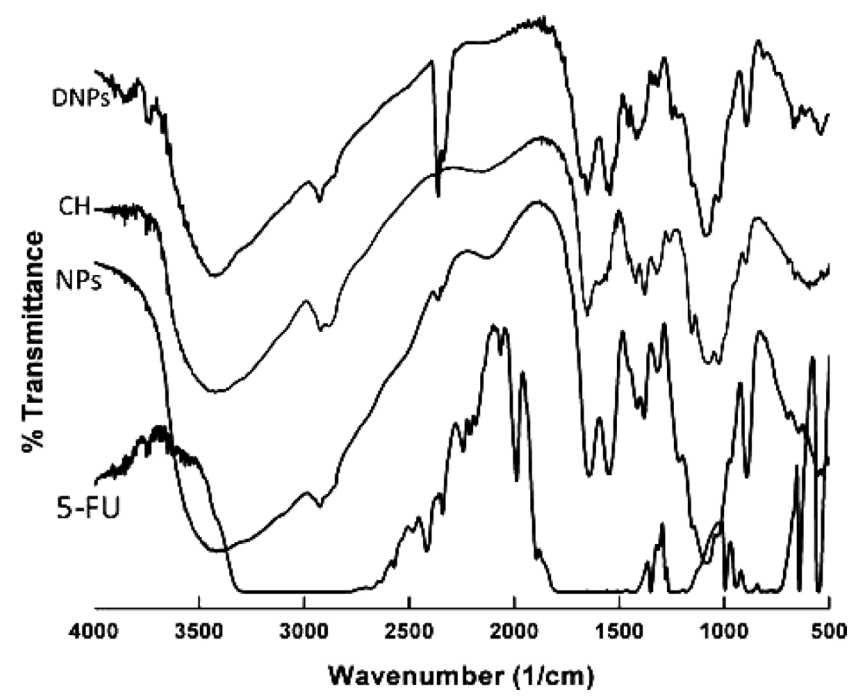

Fig. 6. Comparison of FT-IR Spectra of CH, 5-FU, NPs and DNPs

hydroxyl groups, which remains almost at the same position in blank NPs, and the peak at 3443 becomes sharper in the drug loaded nanoparticles as compared to blank NPs, that shows some interaction between drug and blank nanoparticles. On comparison of the spectra of NPs and DNPs the transmittance peak at $1644 \mathrm{~cm}^{-1}$ shifted to $1648 \mathrm{~cm}^{-1}$ in DNPs possibly due to drug encapsulation in the NPs. The presence of drug in the nanoparticles was confirmed by shift of the transmittance peak from 541 to $552 \mathrm{~cm}^{-1}$ (presence in the drug) that confirmed the aromatic ring of the drug molecule.

XRD spectra of chitosan shows two prominent crystalline peaks at $12(2 \theta)$ and $19.85(2 \theta)$ (Fig. 7). In case of CH-NPs peaks were suppressed that crystallized chitosan converted to amorphous form after cross-linked with TPP. CH-DNPs showed the small peak of 5-FU as compare to 5-FU $[28.83(2 \theta)]$. The intensity of peak was very low as compare to pure 5-FU that shows the presence of drug in NPs as well as crystal form of drug.

In-Vitro Drug Release The in-vitro release study of $\mathrm{CH}$ -

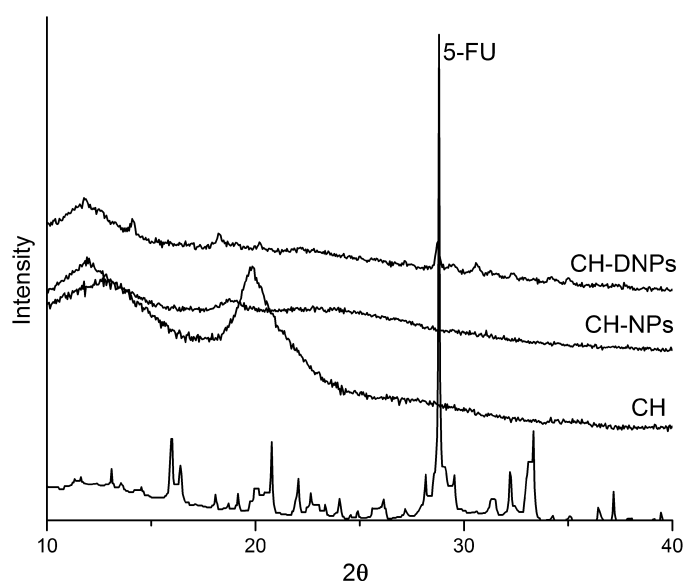

Fig. 7. Comparison of XRD Diffraction Spectra

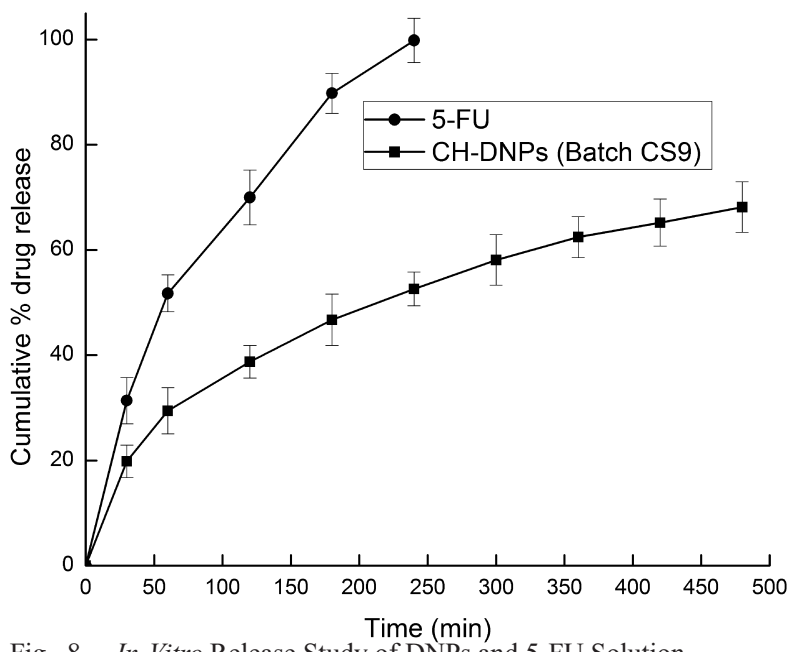

Fig. 8. In-Vitro Release Study of DNPs and 5-FU Solution

DNPs was conducted in phosphate buffer ( $\mathrm{pH} 7.4$ ) for $8 \mathrm{~h}$ and compared with 5-FU solution. The release of 5-FU from the DNPs was sustained manner over a period of $8 \mathrm{~h}$ $(68.15 \%)$ with initial burst release $(30 \%$ in $1 \mathrm{~h})$ (Fig. 8). In case of 5 -FU solution $99.82 \%$ was released within $4 \mathrm{~h}$ with 
burst effect $(51.74 \%$ in $1 \mathrm{~h})$. The results indicated that release of drug from nanoparticles was diffusion-controlled as indicated by higher $r^{2}$ values (0.992) in the Higuchi model. When the release data were analyzed using the KorsmeyerPeppas equation, the $n$-values was 0.439 indicating that drug release from the $\mathrm{CH}$ nanoparticles was Fickian. ${ }^{13)}$

Interaction Study of CH-DNPs with the Mucin The interaction of CH-DNPs with mucin $(0.25,0.12 \% \mathrm{w} / \mathrm{v})$ was studied by measuring the change in viscosity at different time interval (5 to $60 \mathrm{~min}$ ) (Table 2). CH-DNPs increase the viscosity not significantly (one way ANOVA followed by Dunnett's test). Results reveal that no significant interaction was found in between mucin and CH-DNPs. Viscosity change with respect to time was not observed significantly.

In-Vivo Study 5-FU loaded chitosan nanoparticles showed significantly $(p<0.05)$ higher concentration of 5-FU in aqueous humor as compared to 5-FU solution (Fig. 9). The $A U C_{0-8}$ of CH-DNPs and 5-FU solution was 23.85 and 84.5 $\left(\mu \mathrm{g} \cdot \mathrm{ml}^{-1} \cdot \mathrm{h}\right)$ respectively. $C_{\max }$ of $5-\mathrm{FU}$ solution was $6.14 \mu \mathrm{g} / \mathrm{ml}$ that is increased to $16.67 \mu \mathrm{g} / \mathrm{ml}$ in case of $5-\mathrm{FU}$ loaded $\mathrm{CH}$ nanoparticles. $T_{\max }$ of CH-DNPs and 5-FU solution were 2 and $1 \mathrm{~h}$ respectively.

Ocular Tolerability $\mathrm{CH}$ nanoparticles did not show any sign of ocular inflammation or tissue alteration in the rabbit eye in both eye applied nanoparticles and saline as control. The corneal tissue was studied of both eye by slit lamp and

Table 2. In-Vitro Mucin Interaction Study of Mucin and Chitosan Nanoparticles

\begin{tabular}{rcccc}
\hline \hline & \multicolumn{4}{c}{ Viscosity $(\mathrm{cP})$} \\
\cline { 2 - 5 }$(\min )$ & $\begin{array}{c}\text { Mucin } \\
(0.25 \% \mathrm{w} / \mathrm{v})\end{array}$ & $\begin{array}{c}\text { *Mucin } \\
+\mathrm{CH}-\mathrm{DNPs}\end{array}$ & $\begin{array}{c}\text { Mucin } \\
(0.12 \% \mathrm{w} / \mathrm{v})\end{array}$ & $\begin{array}{c}\text { *Mucin } \\
+\mathrm{CH}-\mathrm{DNPs}\end{array}$ \\
\cline { 2 - 5 } & & $4.8 \pm 0.2$ & $2.9 \pm 0.06$ & $2.9 \pm 0.07$ \\
5 & $4.5 \pm 0.4$ & $4.9 \pm 0.2$ & $2.9 \pm 0.05$ & $3.0 \pm 0.05$ \\
10 & $4.5 \pm 0.3$ & $5.2 \pm 0.1$ & $2.8 \pm 0.04$ & $2.9 \pm 0.06$ \\
30 & $4.6 \pm 0.2$ & $5.0 \pm 0.1$ & $2.8 \pm 0.05$ & $2.9 \pm 0.04$ \\
60 & $4.8 \pm 0.1$ & $5.0 \pm 0.2$ & $2.7 \pm 0.03$ & $2.8 \pm 0.04$ \\
\hline
\end{tabular}

* Mucin +CH-DNPs: after dispersion of nanoparticles in mucin.

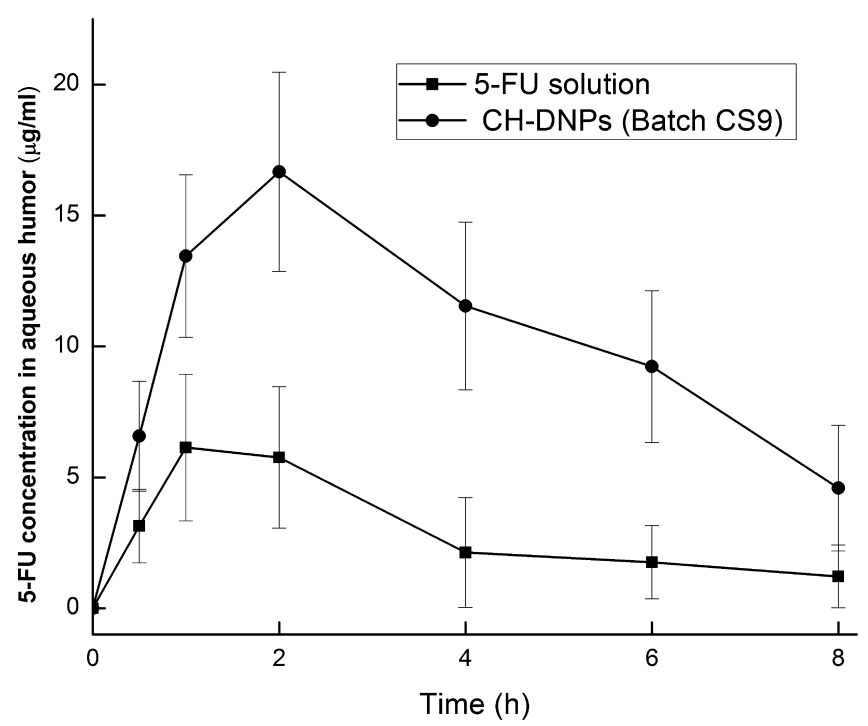

Fig. 9. In-Vivo Study of 5-FU Loaded CH-DNPs and Comparison with 5FU Solution was not found to damage the epithelial layer of cornea and some particles were observed on the corneal surface that confirmed the bioadhesivenes of fabricated NPs. The score of conjuctival congestion, swelling, and discharge were zero at all times of observation (data not shown). The absence of in vivo irritant activity supports for the ocular use of the fabricated NPs.

\section{Discussion}

The 5-FU loaded NPs were prepared to target the ocular surface (conjunctiva/cornea) as nano-sized drug reservoir. Topical application of DNPs can be promising approach to enhance the bioavailability of 5-FU at surface tumor cells because of its nano size and mucoadhesive property. To achieve this aim the nanoparticulate system was fabricated in the size range of $114-197 \mathrm{~nm}$. An optimum size range is required to enhance the bioavailability of 5-FU at ocular surface or disease site. Smaller (100-nm) particles exhibited the highest uptake compared to larger $(800 \mathrm{~nm}$ and $1000 \mathrm{~nm})$ particles and particles of $100 \mathrm{~nm}$ were able to penetrate the corneal barrier. ${ }^{14)}$ Size of the nanoparticles was significantly affected by the different $\mathrm{CH}$ and TPP concentrations, preparation technique and drug loading. The results showed that the size was dependent on morphology, diameter and surface area of particles as well as the measurement of size in different phases like colloidal (dispersion of NPs in a suitable vehicle) or solid phase (lyophilized powder). The particle size and the zeta potential increase linearly with increasing CH-TPP mass ratio. ${ }^{15)}$ The zeta potential was decreased after drug loading due to the anionic nature of 5-FU. It approaches the TPP/CH core through a combination of ionic and hydrogen bonding interactions, driven by electrostatic attraction. ${ }^{4}$ It could be responsible for high drug loading of the $\mathrm{CH}$ nanoparticles because of ionization of 5-FU at high $\mathrm{pH}$ with several possible anionic forms.

In the colloidal dispersion, distribution of particles and the measuring principle of DLS depend on the core of each particle, sometimes clumping of particles may change particle size distribution. The shape of particle depends on TPP and $\mathrm{CH}$ concentration. Particle size range in SEM images was close to DLS data.

The selection of method of preparation was optimized using addition technique such as drop-by-drop method and flush method. The adding procedure of TPP to the $\mathrm{CH}$ solution may also affect the size, shape and zeta potential of nanoparticles. In the first technique, wider size range was obtained as already reported that increasing $\mathrm{CH}$ as well as TPP concentrations will lead to increasing diameter and agglomeration of the produced nanoparticles. ${ }^{10)}$ In addition, the larger sized nanoparticles with higher $\mathrm{CH}$ concentration may also contribute to a higher amount of unneutralized $-\mathrm{NH}^{3+}$ led to making the $\mathrm{CH}$ chain stretch to result in larger nanoparticles. Flush mixing of TPP with $\mathrm{CH}$ solution was found suitable with optimized size range. Higher amounts of TPP with constant mass of $\mathrm{CH}$ could be saturated the cationic sites of the polymeric chain and increase the size of the nanoparticles and may also rise in solution $\mathrm{pH}$, with a consequential effect on increased overall negative surface charge, that can affect the particle size as well as encapsulation of 5-FU. Similar result was also reported by Shu and $\mathrm{Zhu}^{16)}$ and $\mathrm{Hu}$ et al. ${ }^{17)}$ Below $\mathrm{pH} 4.5$, the stronger protona- 
tion of the $\mathrm{NH}^{+2}$ moiety led to a higher zeta potential and a stronger intramolecular repulsion, making the CS chain stretch and resulting in larger nanoparticles. ${ }^{18)}$ However, the protonation of the $\mathrm{NH}^{+2}$ at $\mathrm{pH} 5.5$ in CS molecules is essentially so weak that the zeta potential declined sharply. Zeta potential of CH-TPP nanopaticles decreased with increasing $\mathrm{CH}$ concentration, which may be due to the differences in molecular masses and deacetylation degrees of the $\mathrm{CH}$ used. Increase in $\mathrm{CH}$ concentration also affected the surface charge of nanoparticles because of more unneutralized $-\mathrm{NH}^{3+}$ on the surface of nanoparticles formed.

It was assumed that cationic surface charge of $\mathrm{CH}$ nanoparticles may be interacting with anionic surface of eye. In-vitro mucus interaction study was conducted to measure the change the viscosity of mucus after and before addition of nanoparticles. Significantly, this study was to simulate the tear viscosity change because of nanoparticles. ${ }^{8)}$ It may be due to mucin carboxylic acid groups, which is available in ionized form to interact with the cationic charge of amine groups of chitosan. It was considered that blink process requires low tear viscosity in order to avoid damage to the corneal epithelium. Results reveal that no significant interaction was found.

Increased bioavailability of 5-FU in case of CH-DNPs can be attributed to increased corneal residence time because of mucoadhesive property of chitosan nanoparticles. 5-FU solution showed very low concentration in aqueous humor and some extent was measured in blood that reflects the systemic absorption. In-vitro release data showed ca. 50\% release in $3 \mathrm{~h}$ and $T_{\max }$ in in-vivo study was $2 \mathrm{~h}$. In-vitro sustained release data seem to be in agreement with in-vivo profile of CH-DNPs. Gradual release of 5-FU can maintain the effective concentration anterior chamber of eye for longer duration. No detectable amount of 5-FU was found in blood in case of CH-DNP.

Stored nanosuspension was equally opalescent with only few settled particles in the bottom of the vial that may be due to presence of aggregates of some bigger particles present in the freshly prepared nanosuspension. The $\mathrm{pH}$ increased with time because of drug leaching from the nanoparticles after storing for 1 month at room temperature. Total drug content was not significantly changed in the stored lyophilized powder at $5{ }^{\circ} \mathrm{C}$ but in the nanosuspension more than $50 \%$ drug was present in the suspending medium that suggested that the DNPs cannot be stored in the form of nanosuspension.
Overall result suggested that the freeze dried DNP should be stored in sterile vial. $\mathrm{CH}$ particles undergo volume phase transition (swelling/shrinking processes) upon alteration of $\mathrm{pH}$ from acidic to basic values by a de-swelling process. It is also reported that the diameter of the nanoparticles show a clear tendency to diminish when $\mathrm{pH}$ is increased from 4.0 to 7.0. Swelling and colloidal aggregation practically cause the particles to disintegrate when salt is added, even at molecular concentrations. A swelling mechanism originated by osmotic pressure is associated with ionic distribution between the inner and outer part of the gel. It can also affect the drug loading inside the matrix by modification of diffusion coefficient of the particles. ${ }^{19)}$

Acknowledgments The authors are grateful to University Grants Commission (UGC), New Delhi, and Banaras Hindu University, Varanasi221005, India, for Senior Research Fellowship to Ramesh Chand Nagarwal for supporting this research work.

\section{References}

1) Midena E., Angeli C. D., Valenti M., De Belvis V., Boccato P., Br. J. Ophthalmol., 84, 268-272 (2000).

2) Simeonova M., Velichkova R., Ivanova G., Enchev V., Abrahams I., Int. J. Pharm., 263, 133-140 (2003).

3) Bozkir A., Saka O. M., Il Farmaco, 60, 840-846 (2005).

4) Yang H. C., Hon M. H., Microchem. J., 92, 87-91 (2009).

5) Zheng Y., Wuli Y., Wang C., Fu S., Dong L., Wu L., Eur. J. Pharm. Biopharm., 67, 621-631 (2007).

6) Zhu L., Ma J., Jia N., Zhao Y., Shen H., Colloids Surf. B Biointerfaces, 68, 1-6 (2009).

7) Nagarwal R. C., Kant S., Singh P. N., Maiti P., Pandit J. K., J. Controlled Release, 136, 2-13 (2009).

8) De Campos A. M., Diebold Y., Carvalho E. L. S., Sanchez A., Alonso M. J., Pharm. Res., 21, 803-810 (2004).

9) Lehr C. M., Bowstra J. A., Schacht E. H., Juginger H. E., Int. J. Pharm., 78, 43-48 (1992).

10) Calvo P., Remunan-Ropez C., Vila-Jato J. L., Alonso M. J., J. Appl. Polym. Sci., 63, 125-132 (1997).

11) Alsarra I. A., Alarifi M., J. Chromatogr. B, 804, 435-439 (2004).

12) Mcdonald T. O., Shadduck J. A., "Advance in Modern Toxicology," Vol. 4, ed. by Marzulli F. M., Maibach H. I., Wiley, New York, 1977.

13) Costa C., Lobo C. J. M., Eur. J. Pharm. Sci., 13, 123-133 (2001).

14) Qaddoumi M. G., Ueda H., Yang J., Davda J., Labhasetwar V., Lee V. H. L., Pharm. Res., 21, 641-648 (2004).

15) Gan Q., Wang T., Cochrane C., McCrron P., Colloids Surf. B Biointerfaces, 44, 65-73 (2005).

16) Shu X. Z., Zhu K. J., Int. J. Pharm., 201, 51-58 (2000).

17) Hu B., Pan C., Sun Y., Hou Z., Ye H., Zeng X., J. Agric. Food Chem., 56, 7451-7458 (2008).

18) Zhang L., Kosaraju S. L., Eur. Polym. J., 43, 2956-2966 (2007).

19) Lopez-Leon T., Carvalho E. L. S., Seijo B., Ortega-Vinuesa J. L., Bastos-Gonzalez D., J. Colloid. Interface Sci., 283, $344-351$ (2005). 